\title{
The new frontier in pure autonomic failure: getting a grip on cerebral blood flow
}

\author{
Jurgen A. H. R. Claassen ${ }^{1}$
}

Received: 20 April 2021 / Accepted: 20 April 2021 / Published online: 29 May 2021

○) Springer-Verlag GmbH Germany, part of Springer Nature 2021

Keywords Orthostatic hypotension · Supine hypertension · Transcranial Doppler ultrasound · Brain magnetic resonance imaging

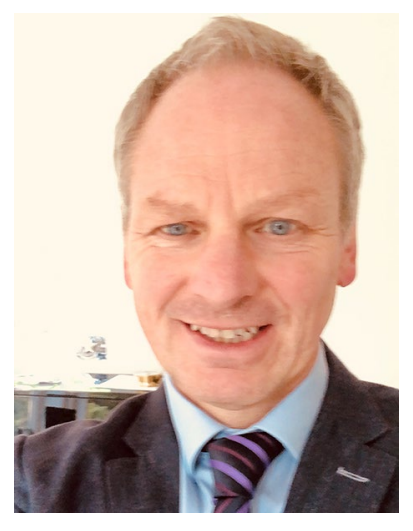

Research into pure autonomic failure (PAF) has largely focused on its effects on blood pressure (BP) and heart rate. However, the most prominent symptoms experienced by patients with PAF, for example dizziness, syncope, and blurred vision, are only indirectly caused by BP. Rather, these symptoms are directly determined by changes in cerebral blood flow (CBF) and oxygen delivery to the brain. While it is undisputed that the large and sudden changes in BP in PAF are the main driver of these changes in CBF, mechanisms such as cerebral autoregulation mediate the relationship between BP and CBF [2]. This means that if, for the same change in BP, some patients experience more symptoms than others, this difference may be explained by how effective autoregulation is at restoring CBF [2]. With

Jurgen A. H. R. Claassen

Jurgen.Claassen@radboudumc.nl

1 Department of Geriatric Medicine, Radboud University Medical Center, Route 925, PO Box 9101, 6500 HB Nijmegen, The Netherlands this in mind, it would make sense to measure changes in $\mathrm{CBF}$, and not only in BP, in patients with PAF and other disorders characterized by efferent baroreflex failure (i.e., neurogenic orthostatic hypotension and supine hypertension). This is rarely done, however.

The limited number of studies on CBF in PAF can be explained, because CBF measurements are technically complex, much more so than BP measurements. They are also much more expensive. When designing a CBF study in patients with PAF, there is the choice between a dynamic method-one that allows measurements of changes in CBF following changes in BP [6] - and a 'static' method that looks at steady-state or baseline differences in CBF between $\mathrm{PAF}$ and controls. If one aims to investigate the effects of postural change in $\mathrm{BP}$ on $\mathrm{CBF}$, a dynamic method is needed. For that purpose, often transcranial Doppler (TCD) is chosen, as it allows measurements of rapid changes (seconds) and can be used with postural changes [1]. TCD however is limited to measurement in one vessel (often the MCA), and measurements of blood velocity can be influenced by changes in vessel diameter. Brain magnetic resonance imaging (MRI) could solve this by measuring global CBF; however, the temporal resolution remains too low to capture the fast changes in CBF that occur in PAF. An important other limitation of MRI is that it is limited to supine conditions, and maneuvers to induce changes in BP (thigh cuff release, or a lower body negative pressure box) cause motion artifacts or are poor proxies of orthostatic changes.

In this issue of Clinical Autonomic Research, Trujillo and colleagues, led by Daniel Claassen (no family relationship with me), have used a 'static' method to investigate baseline changes in patients with PAF [7]. Specifically, the authors applied steady-state CBF measurements using MRI arterial spin labeling (ASL). Their aim was to test the hypothesis that baseline CBF would be lower in PAF. They however 
observed the opposite-a higher CBF in patients with PAF; and in exploratory analyses found more venous hyperintensities; both correlated with higher PAF disease burden (i.e., more evidence of underlying pathology). How can we interpret these unexpected findings?

First, the original hypothesis that CBF would be lower in PAF, although not explicitly explained by the authors, was probably based on the notion that patients with PAF have supine hypertension, and that hypertension is associated with lower CBF [8]. In support of this hypothesis, within the group of PAF patients, higher supine BP was indeed associated with lower CBF in PAF [7]. However, overall, CBF was substantially (on average $10 \mathrm{ml} / \mathrm{min} / 100 \mathrm{~g}$ ) higher in patients with PAF than in controls. This could mean that the negative effects of hypertension on CBF occur as expected in these patients, but at a higher baseline CBF. The question then turns to why patients with PAF would have a higher baseline CBF. To begin with, MRI-ASL is certainly not an optimal method to measure absolute values for CBF. It has a low signal-to-noise-ratio, and absolute values are influenced by measurement technique (e.g., T1 relaxation time, voxel size, inclusion of white matter in the grey matter region of interest). However, controls and patients with PAF were scanned using the same scanner and protocol. This makes artifacts or measurement errors a less likely explanation for this difference. Hematocrit importantly affects CBF (lower hematocrit is associated with higher CBF), but patients with PAF did not have low hematocrit to explain their higher CBF. The higher CBF was not a universal finding, but seemed restricted to about a third of patients with PAF, although this may be an underestimation if we would account for the reduction in CBF in patients with PAF with the highest supine BP. All this suggests that, at least in a subset of patients with PAF, grey matter CBF is significantly higher than in controls, pointing towards cerebral hemodynamic alterations related to PAF.

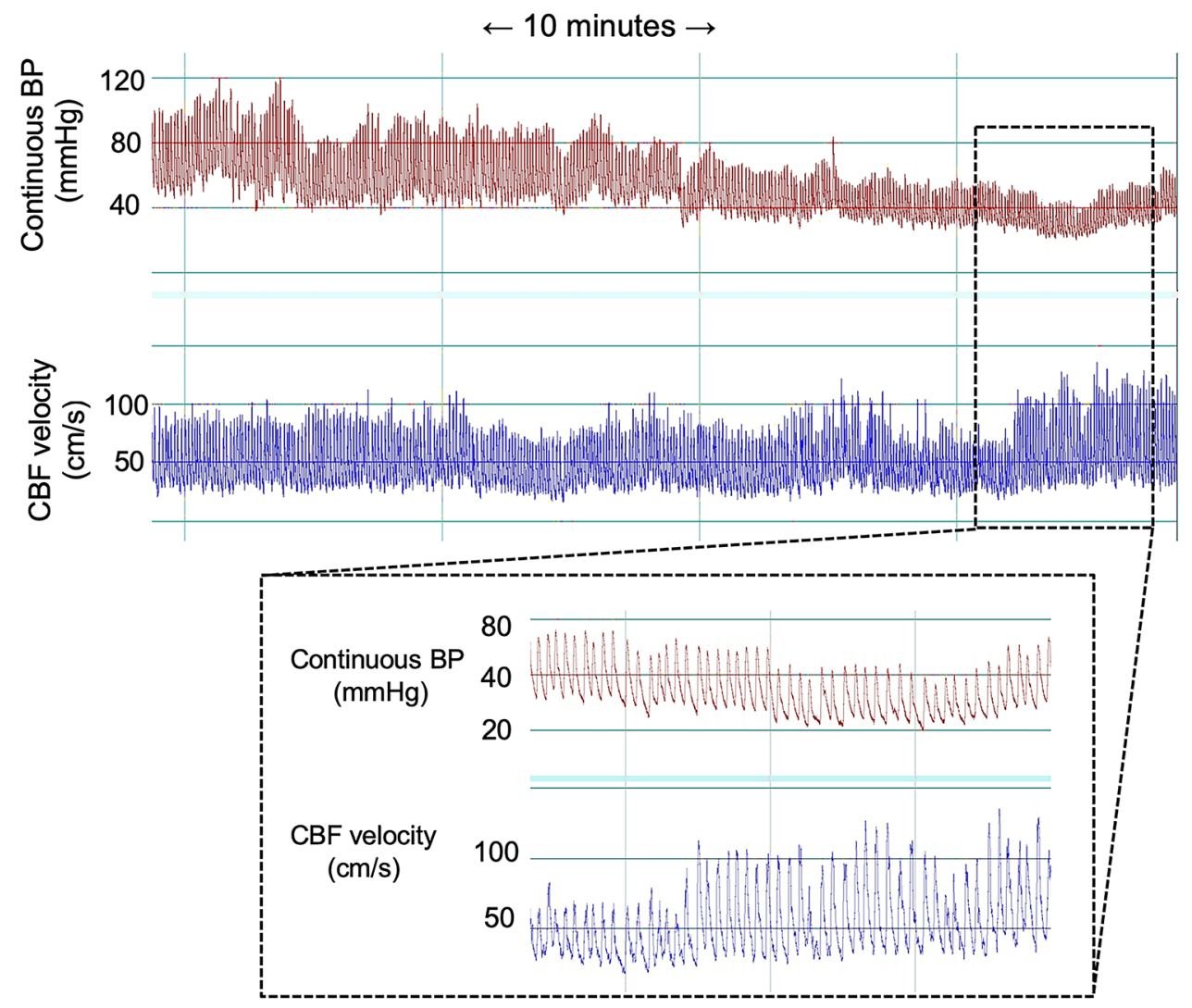

Fig. 1 Continuous blood pressure and cerebral blood flow measurements in a patient with pre-syncope. Continuous recording of blood pressure (BP, Finapres ${ }^{\circledR}$ ) and cerebral blood flow (CBF velocity, transcranial Doppler ultrasound recording of blood velocity in the middle cerebral artery) during a tilt-table test without pharmacological intervention. The patient, a 79-year-old woman, presented to the clinic with frequent episodes of dizziness and falls. Her BP in the supine position was $150 / 80 \mathrm{mmHg}$, with a heart rate of $74 \mathrm{bpm}$ (heart rate not shown). The figure shows a gradual reduction in BP during head-up tilt, reaching values of $\sim 50 / 30 \mathrm{mmHg}$ with a heart rate of $75 \mathrm{bpm}$ (heart rate not shown), when she reported feeling symptoms of pre-syncope such as nausea and sweating, although she did not experience loss of consciousness. The inset illustrates that consciousness was maintained due to a restoration of CBF towards normal values. Note that while systolic CBF velocity returned to near-normal values, her diastolic $\mathrm{CBF}$ velocity remained low. This suggests maximal cerebral vasodilatation due to preserved autoregulation 
The mechanisms that underly these changes cannot be determined from this study. Speculatively, the higher supine CBF may be related to a leftward shift in cerebral autoregulation, towards lower BP values. Most patients with PAF spend most hours of the day walking, upright, with low BP levels. For a sitting or standing systolic BP of $80 \mathrm{mmHg}$, systolic perfusion pressure in the brain is only $\sim 50 \mathrm{mmHg}$. Autoregulation compensates for this through cerebral vasodilation (Fig. 1). A similar phenomenon has been described in patients with congenital afferent baroreflex failure (i.e., familial dysautonomia) [3]. Adaptation to a state that favors cerebral vasodilation improves autoregulation for low BP, at the cost of autoregulation for hypertension. An MRI measurement of $\mathrm{CBF}$ occurs under supine hypertension, which could represent a sudden increase in systolic BP from 80 to $160 \mathrm{mmHg}$, and an increase in cerebral perfusion pressure from 50 to $160 \mathrm{mmHg}$. This may reach the upper limit of the-leftward shifted-autoregulation curve and cause an elevation in CBF. This hypothesis could be tested easily with TCD under postural changes (Fig. 1).

Another possibility is that the increase in CBF reflects a change in sympathetic cerebrovascular innervation. In other words, in addition to the well-characterized peripheral changes, impairing systemic arterial vasoconstriction, the increase in CBF may be mediated by impaired sympathetic autonomic vasoconstriction. Although the autonomic nervous system has only limited direct innervation to cerebral blood vessels (only the proximal large vessels), and is thought to have a minor role in autoregulation, most work suggests that sympathetic cerebral vasoconstriction may protect against hyperperfusion in acute bouts of hypertension [2]. Thus, autonomic failure may reduce the cerebrovascular protection against the sudden large increase in BP from standing to supine in a PAF patient, causing a rise in CBF.

The unexpected finding of venous hyperintensities, although exploratively, supports the idea that the normal physiology of the cerebral circulation is altered in patients with PAF.

Finally, because the elevated CBF may only be present in a subset of patients with PAF, it is possible that this subset reflects patients with an underlying neurodegenerative disease. Indeed, many patients with PAF, on longitudinal follow-up, turn out to have Parkinson disease, dementia with Lewy body, or multiple system atrophy [4]. In patients with Alzheimer's disease, CBF may increase in the early, asymptomatic phase, although probably only in specific regions, but with more advanced disease, global CBF is reduced [5]. Therefore, elevated $\mathrm{CBF}$ in PAF could be a marker for underlying neurodegenerative disease.

Hopefully, the intriguing findings of Trujillo and colleagues will inspire further research to answer all the questions it has raised.

Funding None.

\section{Declarations}

Conflict of interest None.

\section{References}

1. Aaslid R, Lindegaard KF, Sorteberg W, Nornes H (1989) Cerebral autoregulation dynamics in humans. Stroke 20:45-52

2. Claassen JAHR, Thijssen DHJ, Panerai RB, Faraci FM (2021) Regulation of cerebral blood flow in humans: physiology and clinical implications of autoregulation. Physiol Rev. https://doi. org/10.1152/physrev.00022.2020

3. Fuente Mora C, Palma JA, Kaufmann H, Norcliffe-Kaufmann L (2017) Cerebral autoregulation and symptoms of orthostatic hypotension in familial dysautonomia. J Cereb Blood Flow Metab 37:2414-2422

4. Kaufmann H, Norcliffe-Kaufmann L, Palma JA, Biaggioni I, Low PA, Singer W, Goldstein DS, Peltier AC, Shibao CA, Gibbons CH, Freeman R, Robertson D, Autonomic Disorders C (2017) Natural history of pure autonomic failure: A United States prospective cohort. Ann Neurol 81:287-297

5. Kisler K, Nelson AR, Montagne A, Zlokovic BV (2017) Cerebral blood flow regulation and neurovascular dysfunction in Alzheimer disease. Nat Rev Neurosci 18:419-434

6. Panerai RB, Dawson SL, Eames PJ, Potter JF (2001) Cerebral blood flow velocity response to induced and spontaneous sudden changes in arterial blood pressure. Am J Physiol Heart Circ Physiol 280:H2162-2174

7. Trujillo P, Roman OC, Hay KR, Juttukonda MR, Yan Y, Kang H, Paranjape SY, Garland EM, Shibao CA, Biaggioni I, Donahue MJ, Claassen DO (2021) Elevated cerebral blood flow in patients with pure autonomic failure. Clin Auton Res. https://doi.org/10.1007/ s10286-021-00792-8

8. Tryambake D, He J, Firbank MJ, O'Brien JT, Blamire AM, Ford GA (2013) Intensive blood pressure lowering increases cerebral blood flow in older subjects with hypertension. Hypertension 61:1309-1315 